\title{
Direct Somatosensory Projections from the Spinal Cord to the Hypothalamus and Telencephalon
}

\author{
Rami Burstein, Kenneth D. Cliffer, and Glenn J. Giesler, Jr. \\ Department of Cell Biology and Neuroanatomy, University of Minnesota, Minneapolis, Minnesota 55455
}

\begin{abstract}
Somatosensory input to the hypothalamus has been thought to ascend via an indirect, multisynaptic pathway. However, we have antidromically identified nociceptive spinal cord neurons that project directly to the lateral hypothalamus in rats. Retrograde tracers injected into the lateral hypothalamus labeled many spinal neurons bilaterally within the marginal zone, the lateral reticulated area, the lateral spinal nucleus, and the area surrounding the central canal. An anterograde tracer injected into these areas of the spinal cord labeled fibers and terminals in the lateral hypothalamus and, surprisingly, in a number of telencephalic areas. These findings demonstrate a direct somatosensory projection from the spinal cord to the hypothalamus and several telencephalic regions.
\end{abstract}

It is known that a variety of somatosensory stimuli can cause marked changes in the firing of hypothalamic neurons. For example, suckling by an infant, changes in body temperature, and painful stimuli can all induce firing in hypothalamic neurons (Summerlee et al., 1981; Kanosue et al., 1984; Boulant and Dean, 1986). In a large number of studies, a direct input from the spinal cord to the hypothalamus was not seen (Mott, 1895; Bowsher, 1957; Mehler et al., 1960; Mehler, 1969; Boivie, 1979; Craig and Burton, 1985). It is generally believed, therefore, that the afferent pathway for somatosensory information from the spinal cord to the hypothalamus is multisynaptic. However, during an electrophysiological study of the termination of the spinothalamic tract, we found that neurons in the dorsal horn of the spinal cord could be antidromically activated from the lateral hypothalamus. In the present studies, we have physiologically characterized a number of such neurons and used retrograde tracers to examine more completely the distribution of such cells within the spinal gray matter. In addition, we have used an anterograde tracing technique to confirm that the hypothalamus contains axons of spinal cord neurons.

\section{Materials and Methods}

Sprague-Dawley rats were anesthetized with urethane $(1 \mathrm{gm} / \mathrm{kg})$, paralyzed with gallamine triethiode, and artificially ventilated. Core temperature and end-tidal $\mathrm{CO}_{2}$ were monitored and kept within normal limits. Single units were recorded within the lumbar spinal cord using

\footnotetext{
Received Mar. 20, 1987; accepted June 11, 1987.
}

This work was supported by grants BNS84187878 and DA03981. We thank Drs. R. Elde, P. Letourneau, V. Seybold, M. Smithberg, and M. Wessendorf for critical comments on the manuscript and Mr. J. Katter for both his technical assistance and critical comments. We also thank Mr. J. Sedgewick for his excellent photographic work.

Correspondence should be addressed to Glenn J. Giesler, Jr., Department of Cell Biology and Neuroanatomy, 4-135 Jackson Hall, University of Minnesota, Minneapolis, MN 55455.

Copyright (C) 1987 Society for Neuroscience $0270-6474 / 87 / 124159-06 \$ 02.00 / 0$ tungsten microelectrodes. A stainless-steel stimulating electrode was repeatly lowered through rostral portions of the diencephalon in grids of up to 45 tracks separated by $300 \mu \mathrm{m}$. Pulses of $500 \mu \mathrm{A}$ were used to locate axons. Thresholds for antidromic activation were determined at $200 \mu \mathrm{m}$ intervals within each track. At the termination of each experiment, the most anterior low-threshold point and the recording site were marked with small electrolytic lesions. Animals were perfused with $10 \%$ formalin. The brain and spinal cord were removed and cut transversely at $50 \mu \mathrm{m}$ on a freezing microtome. The brains were reacted with the Prussian blue technique. Lesions were reconstructed using a microscope equipped with a camera lucida drawing attachment.

All injections of retrograde tracers were made in rats that were deeply anesthetized with sodium pentobarbital. Tracers were injected stereotaxically into the hypothalamus through a glass micropipette attached to the needle of a microsyringe. The pipette was inserted at a $30^{\circ}$ angle from vertical in an anterior approach. This angled approach was used to avoid passing the pipette through the thalamus. Three retrograde tracers were used in these studies. (1) A $2 \%$ solution of fluoro-gold $(0.1$ $\mu$ l) was injected. Rats survived for $4-7 \mathrm{~d}$ and were perfused with a $4 \%$ paraformaldehyde solution at pH 6.5 , followed by a $4 \%$ paraformaldehyde solution at pH 9.2 (Schmued and Fallon, 1986). Identified spinal segments were cut transversely on a freezing microtome at $40 \mu \mathrm{m}$. Tissue sections were examined using a microscope with ultraviolet epi-illumination. In 2 cases, adjacent sections were carefully analyzed to avoid counting labeled neurons twice. In the remaining cases, alternate sections were analyzed. (2) Injections $(0.1 \mu \mathrm{l})$ of $0.2 \%$ HRP conjugated to wheat germ agglutinin (WGA-HRP) were made. Following a 5-7 d survival, rats were anesthetized and perfused using a solution containing $0.5 \%$ paraformaldehyde and $2.5 \%$ glutaraldehyde (pH 7.6). Sections containing the injection sites were reacted using diaminobenzidine histochemistry (Graham and Karnovsky, 1966). Identified spinal segments were sectioned and reacted using tetramethylbenzidine histochemistry (Mesulam, 1978). (3) A suspension of rhodamine-labeled microspheres $(0.2 \mu \mathrm{l}$ ) was injected (Katz et al., 1984). After surviving 4-5 d, rats were perfused with $4 \%$ paraformaldehyde $(\mathrm{pH} \mathrm{7.4)}$ and the brain and selected spinal segments were sectioned. Reconstructions were made using a microscope equipped with a camera lucida drawing attachment.

Phaseolus vulgaris leucoagglutinin (PHA-L) was used for anterograde tracing. Rats were deeply anesthetized and a laminectomy was performed over either the lumbar or cervical enlargement. Iontophoretic injections of a $2.5 \%$ solution of PHA-L (Vector Labs) in buffered saline (pH 7.2) were made using micropipettes with tip diameters of 10-15 $\mu \mathrm{m}$. A pulsed positive current totaling $40 \mu \mathrm{A}-\mathrm{min}$ was used at each site to eject PHA-L. Attempts were made to inject all areas of the spinal cord in which neurons had been labeled after injections of retrograde tracers into the hypothalamus. Therefore, 8-10 injections were made in each rostrocaudal plane of the cord. From 1-4 planes were injected. Planes were separated by $500 \mu \mathrm{m}$. Rats survived 3-5 weeks and were then perfused with buffered $4 \%$ paraformaldehyde at $\mathrm{pH} 6.5$ followed by buffered $4 \%$ paraformaldehyde at $\mathrm{pH} 9.2$. Sections were cut at 20 $\mu \mathrm{m}$ and immunohistochemically stained using a primary antiserum to PHA-L, a biotinylated secondary antiserum, and fluoresceinated avidin (all from Vector Labs). Sections were counterstained with ethidium bromide.

\section{Results and Discussion}

In the initial studies, we antidromically activated 6 spinal cord neurons from the contralateral ventrobasal complex (VBC) of the thalamus in rats. We then used antidromic activation tech- 

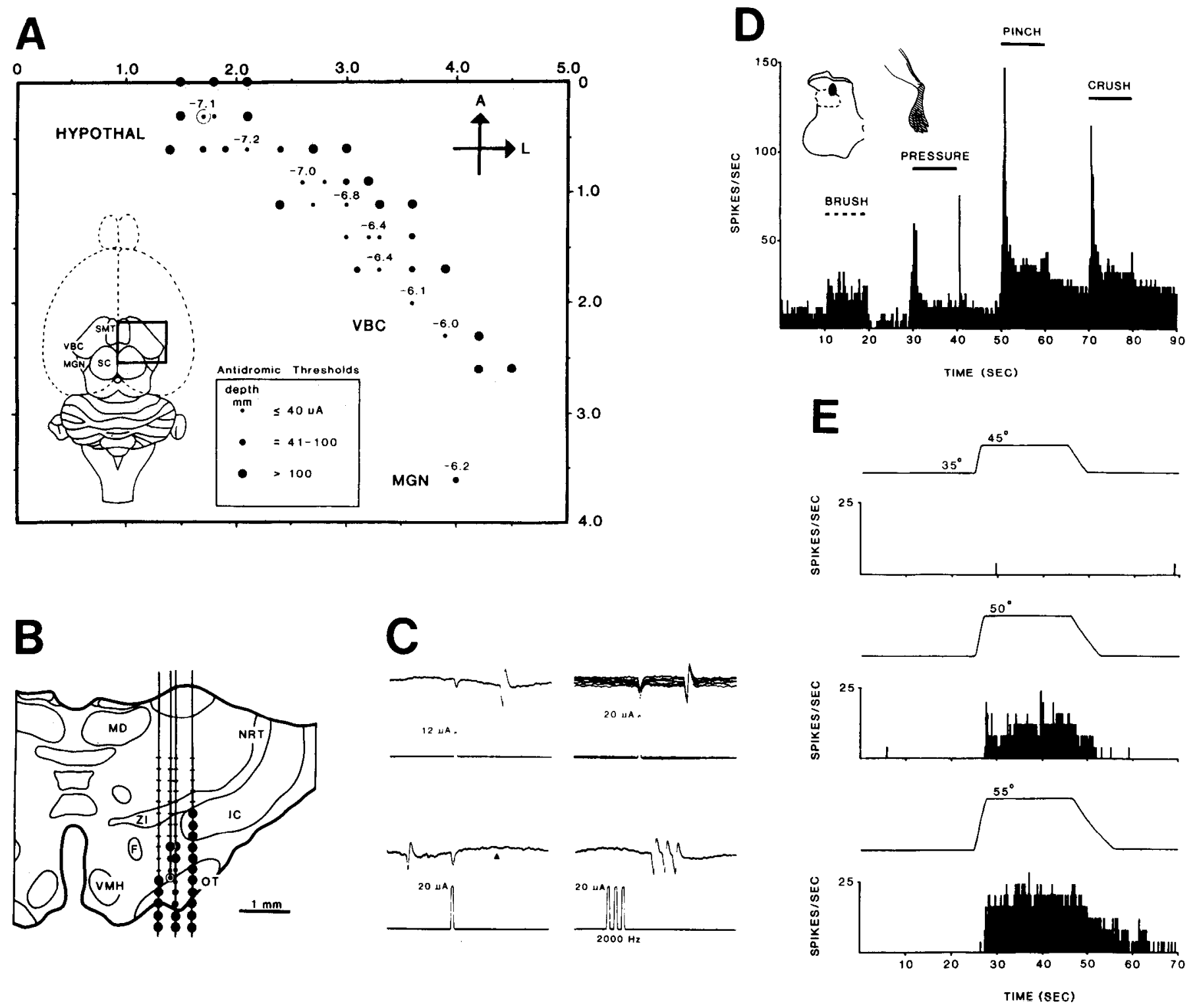

Figure 1. Characterization of a spinohypothalamic tract neuron. $A$, Large square represents a dorsal view of the brain. The position of the square is indicated in the drawing of the brain at left. Within the square, the positions are illustrated of 37 penetrations through the diencephalon with an antidromic stimulating electrode. The distance caudal to bregma is indicated at right and the distance from the midline is indicated at top. The size of each dot is related to the minimum level of current required to activate the neuron within the track (inset). The depth below the cortical surface of the point with the lowest threshold in each anterior-posterior plane is indicated. The axon was followed to progressively more anterior, medial, and ventral positions within the diencephalon. The location of the most anterior track in which a small antidromic threshold was encountered is circled. $A$, anterior; $L$, lateral, $M G N$, medial geniculate nucleus; $S C$, superior colliculus; $S M T$, stria medullaris thalami, $V B C$, ventrobasal complex. $B$, Location in transverse section of the tract circled in $A$. Each penetration of the stimulating electrode is indicated by a vertical line. The sizes of the dots on the vertical lines reflect the same antidromic threshold values as in $A$. Dashes on the vertical lines indicate positions at which antidromic activation could not be produced using $500 \mu \mathrm{A}$ pulses. The position of the point at which the threshold was lowest in this plane is circled. $F$, fornix; $I C$, internal capsulc; $M D$, mcdial dorsal nuclcus; $N R T$, nucleus reticularis thalami; $O T$, optic tract; $V M H$, ventral medial hypothalamic nucleus; $Z I$, zona inserta. $C$, Antidromic responses of the neuron to stimulation at the low-threshold point illustrated in $A$ and $B$. In each panel, the intensity of the antidromic stimulus is indicated in the lower record. The latency of the response was 4.2 msec. Upper left, Response of the neuron to a single $12 \mu \mathrm{A}$ shock in the lateral hypothalamus. Upper right, Overlapping traces of responses to 6 shocks in the hypothalamus; note the stable latency of the response. Lower left, Collision of an orthodromic spike with the antidromic spike. The point at which the antidromic response would have occurred is indicated by an arrowhead. Lower right, Responses of the neuron to a train of 3 pulses delivered at 2000 pulses/sec. $D$, Peristimulustime histogram (bin width, $250 \mathrm{msec}$ ) of the response of the unit to mechanical stimulation within its peripheral receptive field (insert). The period of each stimulus is indicated by a line above the histogram. Note that the largest responses were produced by noxious stimuli. A drawing of the lesion made to mark the recording site within the lumbar dorsal horn is at upper left. $E$, Responses of the unit to thermal stimulation of its receptive field. The period and intensity of each stimulus are indicated in the record above the histogram. Note the increasing responses to increasingly intense noxious thermal stimuli. 
niques to explore the diencephalon for projections of these axons outside the VBC. Five of the 6 neurons antidromically activated from the VBC were found to have axons that could be followed toward or into the hypothalamus. Antidromic thresholds were $<40 \mu \mathrm{A}$. Two additional neurons were antidromically activated from electrodes in the lateral hypothalamus exclusively. In these cases, antidromic thresholds were $<50 \mu \mathrm{A}$. The recording locations of all antidromically activated neurons were located within the marginal zone, nucleus proprius, or lateral reticulated area of the dorsal horn.

Results obtained from a neuron that was antidromically activated from the hypothalamus are illustrated in Figure 1. Figure 11 depicts the locations of 37 penetrations with an antidromic stimulating electrode into the diencephalon. The inset drawing shows the relationship of the grid of penetrations to the dorsal surface of the diencephalon. The size of each dot is related to the lowest current capable of activating the neuron from any point within the track. The depth below the cortical surface at which the threshold was lowest in each anterior-posterior plane is also indicated. Antidromic thresholds were determined at more than 600 points. It is clear from this figure that the axon was followed to progessively more anterior, medial, and ventral positions within the diencephalon. The most anterior point (circled in Fig. $1 A$ ) at which a small current activated the neuron is illustrated in a transverse section through the diencephalon in Figure $1 B$ (circled dot). This low threshold point is located within the lateral hypothalamus.

The antidromic response of this neuron to stimulation in the lateral hypothalamus is demonstrated in Figure $1 \mathrm{C}$. Like the other antidromically activated neurons, this unit responded at a constant latency (upper right) and followed high-frequency stimulus trains (lower right). Also, orthodromic and antidromic spikes collided within a critical interval (lower left). The antidromic threshold at this point was less than $12 \mu \mathrm{A}$ (Fig. $1 C$, upper left). It is likely that this stimulation point was close to the axon; the effective spread of a $12 \mu \mathrm{A}$ current pulse is lcss than $120 \mu \mathrm{m}$ (Abzug et al., 1974; Ranck, 1975). The point was surrounded medially, laterally, dorsally, ventrally, and anteriorly by locations at which considerably more current was required to activate the neuron (Fig. 1, $A$ and $B$ ). These findings suggest that this low threshold point was near the terminal region of the axon.

The neuron was recorded within the lumbar dorsal horn (Fig. $1 D$, inset). It responded to innocuous mechanical stimuli but was more strongly activated by noxious mechanical stimuli (Fig. $1 D$ ) within its receptive field (inset). It was therefore classified as a wide dynamic range (WDR) neuron. It also responded incrementally to noxious thermal stimuli (Fig. 1E).

Six of the 7 recorded cells were classified as WDR neurons. The remaining neuron responded only to noxious stimuli. All examined neurons responded incrementally to increasingly intense noxious thermal stimuli. The incrementing responses of these neurons to graded noxious thermal stimuli strongly suggest that these neurons receive an input that originates in primary afferent nociceptors; nociceptors are the only type of primary afferent fiber capable of responding incrementally to increasingly intense noxious thermal stimuli (Fitzgerald and Lynn, 1977; LaMotte and Campbell, 1978; Hallin et al., 1981).

Retrograde tracing techniques were used to determine more completely the locations of spinal cord neurons that project to the hypothalamus. Nine injections of retrograde tracers that were restricted to the lateral hypothalamus (6 with fluoro-gold, 2 with labeled microspheres, and 1 with WGA-HRP) labeled many neurons in the spinal cord. The injected tracer did not spread into the thalamus or cortex in any of these cases. An example of one such injection is illustrated in Figure $2 A$. This injection of fluoro-gold labeled a large number of neurons bilaterally throughout the length of the spinal cord (Fig. 2A). Approximately half of the labeled neurons were located in the lateral reticulated area. Roughly $10 \%$ of the labeled neurons were found in the gray matter surrounding the central canal. An additional $10 \%$ were found in the marginal zone. These areas are all thought to be importantly involved in nociceptive processing in the spinal cord. All 3 areas are known to receive direct inputs from primary afferent nociceptors (Light and Perl, 1979; Mense et al., 1981; Rethelyi et al., 1982; Sugiura et al., 1986), to contain large percentages of nociceptive second-order neurons (Hillman and Wall, 1969; Christensen and Perl, 1970; Nahin et al., 1983; Honda and Perl, 1985), and to contribute axons to other ascending nociceptive pathways (Giesler et al., 1979; Menetrey et al., 1980, 1982; Kevetter and Willis, 1983).

Twenty-five percent of the retrogradely labeled neurons were found in the lateral spinal nucleus. The function of this nucleus is not well understood (Menetrey et al., 1980, 1982; Giesler and Elde, 1985).

The distribution within the spinal cord of the cells of origin of what we will call the spinohypothalamic tract (SHT) is in some ways similar to the distribution of spinothalamic tract (STT) neurons. For example, many of the cells of origin of both tracts are located within the lateral reticulated area (Giesler et al., 1979; Kevetter and Willis, 1983). However, the distributions of the cells of origin of these 2 tracts also differ in several ways. For example, approximately $40 \%$ of SHT neurons project ipsilaterally, whereas fewer than $10 \%$ of STT neurons project ipsilaterally (Giesler et al., 1979; Kevetter and Willis, 1983). Also, the lateral spinal nucleus and the neurons adjacent to the central canal frequently project to the hypothalamus but do not contribute prominently to the spinothalamic tract (Giesler et al., 1979; Kevetter and Willis, 1983).

The projections of spinal cord neurons within the hypothalamus were examined using the anterograde transport of PHAL. PHA-L is a sensitive anterograde tracer that reveals the detailed morphology of labeled axons and terminals (Gerfen and Sawchenko, 1984). PHA-L was iontophoretically injected unilaterally into the lateral reticular area, the marginal zone, the area around the central canal, and the lateral spinal nucleus of the cervical enlargement. Examples of fibers labeled with PHA-L are illustrated in Figure 2, $B-E$. Many labeled fibers and varicosities were seen bilaterally in the lateral hypothalamus (Fig. $2 B$ ); a few were also seen in the medial hypothalamus. Unexpectedly, additional labeled fibers and varicosities were encountered within a number of nuclei in the basal forebrain and other telencephalic areas. For example, labeled fibers and terminals were seen contralaterally in the nucleus of the vertical limb of the diagonal band of Broca (Fig. $2 C$ ), bilaterally in the medial septal nuclei (Fig. 2D), and ipsilaterally in the medial nucleus accumbens (Fig. $2 E$ ). Frequently, labeled varicosities in all of these areas could be seen in apparent contact with counterstained neurons (Fig. 2, $B-E$ ). Labeled decussating axons were also observed in the supraoptic decussation, including the caudalmost portion of the optic chiasm.

The 3 independent techniques used in this study demonstrate 
A
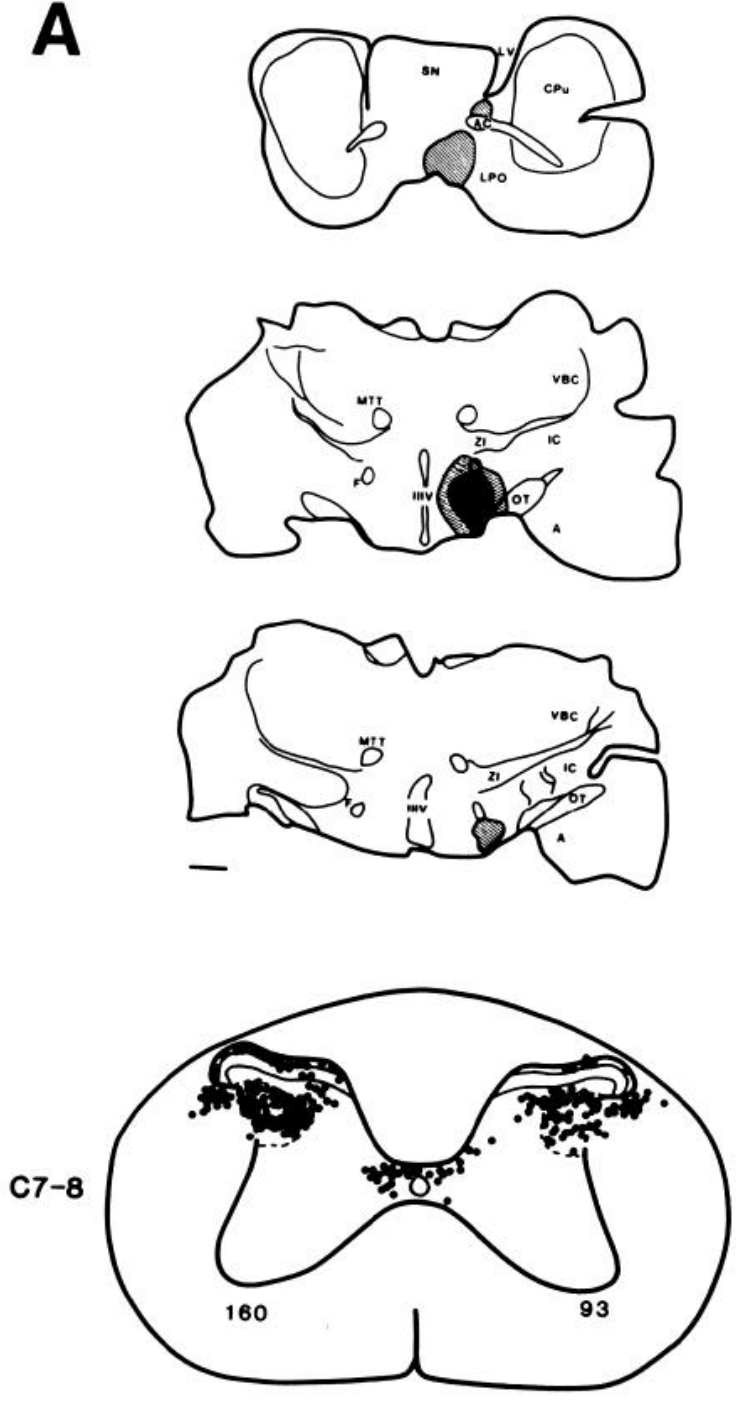

T6
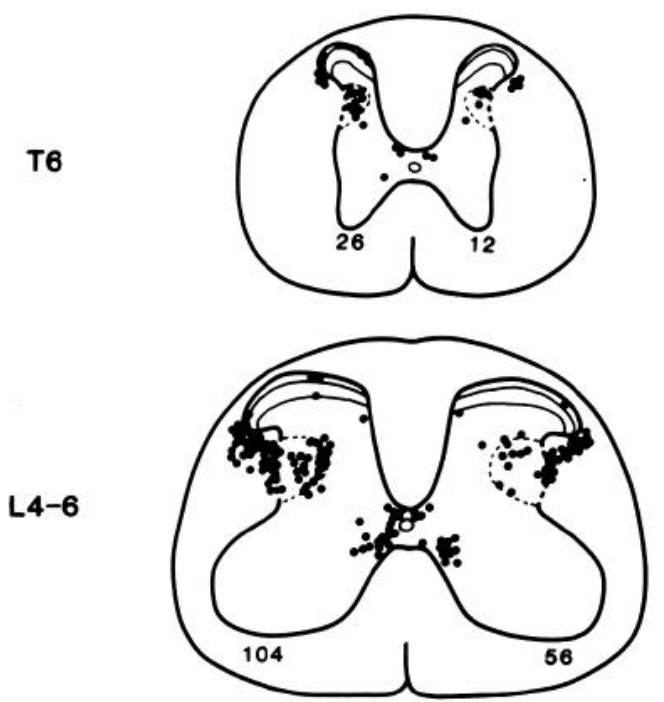
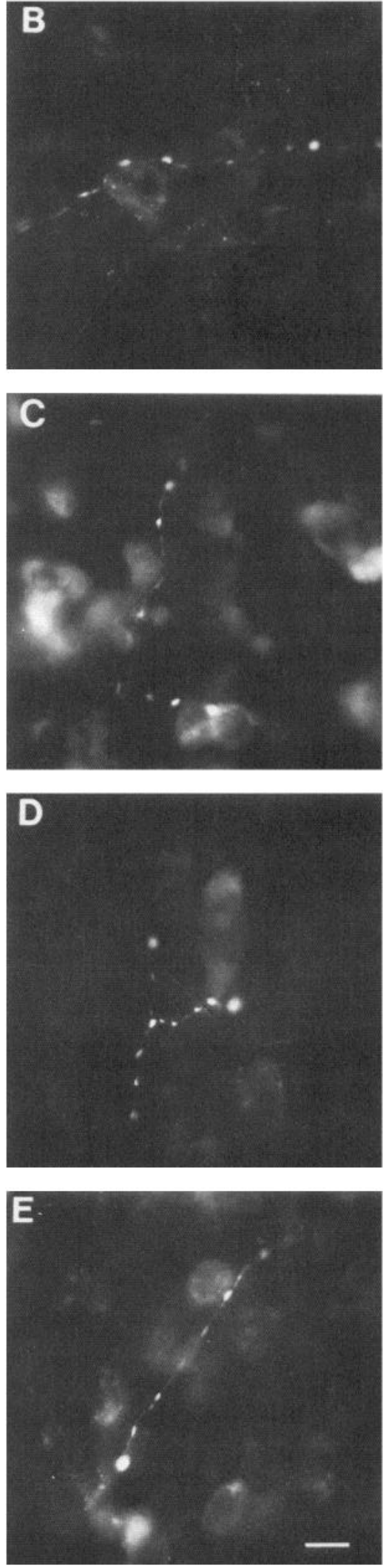

Figure 2. A, Locations of cells of origin of the spinohypothalamic tract. Injection of fluoro-gold into the hypothalamus labeled a large number of neurons bilaterally throughout the length of the cord. The rostral (top) and caudal (bottom) limits and the center of the injection site are depicted. Scale bar, $1 \mathrm{~mm}$. The total number of labeled neurons in each side of the cord within the illustrated segments are indicated. $L V$, lateral ventricle; $S N$, septal nucleus; $C P U$, caudate putamen; $A C$, anterior commissure; $L P O$, lateral preoptic area; $M T T$, mamillothalamic tract; $V B C$, ventrobasal complex; $I C$, internal capsule; $Z I$,zona inserta; $I I I V$, third ventricle; $O T$, optic tract; $A$, amygdala; $F$, fornix. $B-E$, Fibers and terminals in the hypothalamus and telencephalon labeled with PHA-L following unilateral injections into the cervical enlargement of the spinal cord. $B$, Contralateral 
that a direct projection carries somatosensory information from the spinal cord to the hypothalamus in the rat. It appears that an SHT is also present in other species. Ring and Ganchrow (1983) reported that spinal lesions produced degeneration in the lateral hypothalamus of hedgehogs. Ju (1984) found that injections of HRP into the spinal cord of rabbits produced what he interpreted to be anterograde labeling in the hypothalamus. Anderson and Berry (1959) noted that spinal lesions in cats caused degeneration of fibers in the lateral hypothalamus. Although not mentioned in the text of the paper, several of the reconstructions in a paper by Chang and Ruch (1947) appear to show degenerating axons in the supraoptic decussation following spinal lesions in monkeys. Kerr (1975) reported that lesions of the ventral funiculus produced terminal degeneration in the medial hypothalamus in monkeys.

Several telencephalic areas also appear to receive direct projections from spinal cord neurons. Injections of PHA-L into the spinal cord gray matter labeled axons within the basal forebrain, septal nuclei, and nucleus accumbens. To determine the origin of these projections, we (Burstein, Cliffer and Giesler, unpublished observations) have recently injected fluoro-gold into each of these telencephalic areas. In each case, spinal cord neurons were labeled bilaterally in nucleus proprius, marginal zone, lateral spinal nucleus, and the area around the central canal. These injections labeled neurons in all examined segments. These findings indicate that neurons in several areas of the spinal cord gray matter at all segmental levels of the cord project to a number of telencephalic regions. To our knowledge, such projections have not been previously reported.

The lateral hypothalamus, septal nucleus, and nucleus accumbens are all bclicved to participatc in the expression of a large number of emotional behaviors including rage and aggressive responses (Panksepp, $1971 \mathrm{a}, \mathrm{b}$; Blanchard et al., 1977; Albert et al., 1982). It will be important to determine whether direct spinal nociceptive projections to these areas play a role in the production of such responses to nociceptive stimulation.

\section{References}

Abzug, C., M. Maeda, B. W. Peterson, and V. J. Wilson (1974) Cervical branching of lumbar vestibulospinal axons. J. Physiol. (Lond.) 243: 499-522.

Albert, D. J., M. L. Walsh, J. Ryan, and Y. Siemens (1982) Mouse killing in rats: A comparison of spontaneous killers and rats with lesions of the medial hypothalamus or the medial accumbens nucleus. Physiol. Behav. 29: 989-994.

Anderson, F. D., and C. M. Berry (1959) Degeneration studies of long ascending fiber systems in the cat brain stem. J. Comp. Neurol. 111: 195-230.

Blanchard, D. C., R. J. Blanchard, L. K. Takahashi, and T. Takahashi (1977) Septal lesion and aggressive behavior. Behav. Biol. 21: 157161.

Boivie, J. (1979) An anatomical reinvestigation of the termination of the spinothalamic tract in the monkey. J. Comp. Neurol. 186: 343370 .

Boulant, J. A., and J. B. Dean (1986) Temperature receptors in the central nervous system. Annu. Rev. Physiol. 48: 639-654.

Bowsher, D. (1957) Termination of the central pain pathway in man: The conscious appreciation of pain. Brain 80:606-621.

Chang, H. T., and T. C. Ruch (1947) Topographical distribution of spinothalamic fibers in the thalamus of the spider monkey. J. Anat. (Lond.) 81: 150-164.

Christensen, B. N., and E. R. Perl (1970) Spinal neurons specifically excited by noxious or thermal stimuli: Marginal zone of the dorsal horn. J. Neurophysiol. 33: 293-307.

Craig, A. D., and H. Burton (1985) The distribution and topographical organization in the thalamus of anterogradely-transported horseradish peroxidase after spinal injections in the cat and raccoon. Exp. Brain Res. 58: 227-254.

Fitzgerald, M., and B. Lynn (1977) The sensitization of high threshold mechanoreceptors with myelinated axons by repeated heating. J. Physiol. (Lond.) 365: 549-563.

Gerfen, C. R., and Sawchenko, P. (1984) An anterograde neuroanatomical tracing method that shows the detailed morphology of neurons, their axons and terminals: Immunohistochemical localization of an axonally transported plant lectin, phaseolus vulgaris leucoagglutinin (PHA-L). Brain Res. 290: 219-238.

Giesler, G. J. Jr., and R. P. Elde (1985) Immunohistochemical studies of the peptidergic content of fibers amd terminals within the lateral spinal and lateral cervical nuclei. J. Neurosci. 5: 1833-1844.

Giesler, G. J. Jr., D. Menetrey, and A. I. Basbaum (1979) Differential origins of spinothalamic tract projections to medial and lateral thalamus in the rat. J. Comp. Neurol. 184: 107-126.

Graham, R. C., and M. J. Karnovsky (1966) The early stages of absorption of injected horseradish peroxidase in the proximal tubules of mouse kidney: Ultrastructural cytochemistry by a new technique. J. Histochem. Cytochem. 11: 291-302.

Hallin, R. G., H. E. Torebjork, and Z. Wiesenfeld (1981) Nociceptors and warm receptor innervated by $\mathrm{C}$ fibers in human skin. I. Neurol. Neurosurg. Psychiatry 44: 313-319.

Hillman, P., and P. D. Wall (1969) Inhibitory and excitatory factors influencing the receptive fields of lamina V spinal cord cells. Exp. Brain Res. 9: 284-306.

Honda, C. N., and E. R. Perl (1985) Functional and morphological features of neurons in the midline region of the caudal spinal cord of the cat. Brain Res. 340: 285-295.

Ju, G. (1984) Direct connections between hypothalamus and lumbar spinal cord in rabbits. Sci. Sin. 2\%:/89-/99.

Kanosue, K., T. Nakayama, Y. Ishikawa, and K. Imai-Matsumura (1984) Responses of hypothalamic and thalamic neurons to noxious and scrotal thermal stimulations in rats. J. Therm. Biol. 9: 11-13.

Katz, L. C., A. Burkhalter, and W. J. Dreyer (1984) Fluorescent latex microspheres as a retrograde neuronal marker for in vivo and in vitro studies of visual cortex. Nature 310:498-450.

Kerr, F. W. L. (1975) The ventral spinothalamic tract and other ascending systems of the ventral funiculus of the spinal cord. J. Comp. Neurol. 159: 335-356.

Kevetter, G. A., and W. D. Willis (1983) Collaterals of spinothalamic cells in the rat. J. Comp. Neurol. 215: 453-464.

LaMotte, R. H., and J. N. Campbell (1978) Comparison of responses of warm and nociceptive C-fibers in monkey with human judgment of thermal pain. J. Neurophysiol. 41: 509-529.

Light, A. R., and E. R. Perl (1979) Spinal termination of functionally identified primary afferent neurons with slowly conducting myelinated fibers. J. Comp. Neurol. 186: 133-150.

Mehler, W. R. (1969) Some neurological species differences-A posteriory. Ann. NY Acad. Sci. 167: 424-468.

Mehler, W. R., M. E. Feferman, and W. J. H. Nauta (1960) Ascending axon degeneration following anterolateral cordotomy. An experimental study in the monkey. Brain $83: 718-750$.

Menetrey, D., A. Chaouch, and J. M. Besson (1980) Location and properties of dorsal horn neurons at origin of spinoreticular tract in lumbar cnlargement of the rat. J. Ncurophysiol. 44: 862-877.

Menetrey, D., A. Chaouch, D. Binder, and J. M. Besson (1982) The origin of the spinomesencephalic tract in the rat: An anatomical study using the retrograde transport of horseradish peroxidase. J. Comp. Neurol. 206: 193-207.

Mense, S., A. R. Light, and E. R. Perl (1981) Spinal terminations of

lateral hypothalamus. $C$, Contralateral nucleus of the vertical limb of the diagonal band of Broca. $D$, Ipsilateral medial septal nucleus adjacent to midline. $E$, Ipsilateral nucleus accumbens. Note that many axons have labeled varicosities that are apposed to counterstained cell bodies. Scale bar, $10 \mu \mathrm{m}$. 
subcutaneous high-threshold mechanoreceptors. In Spinal Cord Sensation: Sensory Processing in the Dorsal Horn, A. G. Brown and M. Rethelyi, eds., pp. 79-88, Scottish Academic Press, Edinburgh.

Mesulam, M. M. (1978) Tetramethyl benzidine peroxidase neurohystochemistry: A noncarcinogenic blue reaction product with superior sensitivity for visualizing neural afferents and efferents. J. Histochem. Cytochem. 26: 106-117.

Mott, F. W. (1897) Experimental enquiry upon the afferent tracts of the central nervous system of the monkey. Brain 18: 1-20.

Nahin, K. L., A. M. Madsen, and G. J. Giesler, Jr. (1983) Anatomical and physiological studies of the gray matter surrounding the spinal cord central canal. J. Comp. Neurol. 220: 321-335.

Panksepp, J. (1971a) Effects of hypothalamic lesions on mouse killing and shock-induced fighting in rats. Physiol. Behav. 6: 311-316.

Panksepp, J. (1971 h) Aggression elicited hy electrical stimulation of the hypothalamus in albino rats. Physiol. Behav. 6: 321-329.

Ranck, J. B. (1975) Which elements are excited in electrical stimulation of mammalian central nervous system: A review. Brain Res. 98: 417 440.
Rethelyi, M., A. R. Light, and E. R. Perl (1982) Synaptic complexes formed by functionally defined primary afferent units with fine myelinated fibers. J. Comp. Neurol. 207: 381-393.

Ring, G., and D. Ganchrow (1983) Projections of nucleus caudalis and spinal cord to brainstem and diencephalon in the hedgehog (Erinaceus europaeus and Paraechinus aethiopicus): A degeneration study. J. Comp. Neurol. 216: 132-151.

Schmued, L. C., and J. H. Fallon (1986) Fluoro-Gold: A new fluorescent retrograde axonal tracer with numerous unique properties. Brain Res. 377: 147-154.

Sugiura, Y., C. L. Lee, and E. R. Perl (1986) Central projections of identified unmyelinated (C) afferent fibers innervating mammalian skin. Science 234: 358-361.

Summerlee, A. J. S., and D. W. Lincoln (1981) Electrophysiological recording from oxytocinergic neurons during suckling in the unanaesthetized lactating rat. J. Endocrinol. 90: 255-265. 